The Annals of Probability

2002, Vol. 30, No. 4, 1959-1976

\title{
ENTROPY INEQUALITIES FOR UNBOUNDED SPIN SYSTEMS
}

\author{
By Paolo Dai Pra, ${ }^{1}$ Anna Maria Paganoni ${ }^{2}$ And Gustavo Posta ${ }^{1}$ \\ Politecnico di Milano
}

\begin{abstract}
We consider nonconservative, reversible spin systems, with unbounded discrete spins. We show that for a class of these dynamics in a high temperature regime, the relative entropy with respect to the equilibrium distribution decays exponentially in time, although the logarithmic-Sobolev inequality fails. To this end we prove a weaker modification of the logarithmic-Sobolev inequality.
\end{abstract}

1. Introduction. In this paper we consider nonconservative spin systems in a finite lattice $\Lambda \subset \mathbf{Z}^{d}$, whose spins take values in $\mathbf{Z}_{+}$; in the particle interpretation, this means that every site of the lattice may be occupied by any number of particles. The dynamics are required to satisfy detailed balance with respect to a (finite volume) Gibbs measure given by, for $\sigma=\left(\sigma_{x}\right)_{x \in \Lambda} \in \mathbf{Z}_{+}^{\Lambda}$,

$$
\mu_{\Lambda}^{\tau}(\sigma)=\frac{1}{Z_{\Lambda}^{\tau}} e^{-\beta H_{\Lambda}^{\tau}(\sigma)} \prod_{x \in \Lambda} \rho\left(\sigma_{x}\right)
$$

where $\rho(\cdot)$ is a given (reference) measure on $\mathbf{Z}_{+}, H_{\Lambda}^{\tau}$ is the Hamiltonian associated to a bounded, finite range interaction, $\tau$ is a fixed configuration in $\mathbf{Z}_{+}^{\mathbf{Z}^{d} \backslash \Lambda}$ that contributes, as boundary condition, to the Hamiltonian $H_{\Lambda}^{\tau}, \beta>0$ is the inverse temperature and $Z_{\Lambda}^{\tau}$ is the normalization factor. We denote by $\mathcal{L}_{\Lambda}^{\tau}$ the infinitesimal generator of one of these dynamics, which will be defined in Section 2.

If the volume $\Lambda$ and the boundary condition $\tau$ are kept fixed, the dynamics we consider have $\mu_{\Lambda}^{\tau}$ as the unique invariant (reversible) measure, and convergence to equilibrium occurs from any initial distribution. The main objective of this paper is to show that, for certain choices of $\rho$ and for $\beta$ sufficiently small, the system converges to equilibrium at an exponential rate which is uniform in $\Lambda$ and $\tau$. In particular, this implies exponential convergence to equilibrium for the limiting infinite volume dynamics on $\mathbf{Z}_{+}^{\mathbf{Z}^{d}}$.

For reversible, nonconservative spin systems whose spins take values on either a finite set [13, 10,9] or a compact manifold [14], uniform convergence to equilibrium is rather well understood. The rate of convergence to equilibrium is typically given in terms of two constants: the spectral gap $\operatorname{gap}\left(\mathcal{L}_{\Lambda}^{\tau}\right)$ and the

Received August 2000; revised November 2001.

${ }^{1}$ Supported in part by MURST project Stochastic processes with spatial structure and GNAFA.

${ }^{2}$ Supported in part by GNAFA.

AMS 2000 subject classifications. 60K35, 82C22.

Key words and phrases. Spin systems, entropy, logarithmic-Sobolev inequality, spectral gap. 
logarithmic-Sobolev constant $S\left(\mathcal{L}_{\Lambda}^{\tau}\right)$ that are defined as the optimal constants of, respectively, the Poincaré inequality,

$$
\mu_{\Lambda}^{\tau}(f, f) \leq-l^{-1} \mu_{\Lambda}^{\tau}\left(f \mathcal{L}_{\Lambda}^{\tau} f\right)
$$

and the logarithmic-Sobolev inequality

$$
\operatorname{Ent}_{\mu_{\Lambda}^{\tau}}\left(f^{2}\right) \leq-\alpha^{-1} \mu_{\Lambda}^{\tau}\left(f \mathcal{L}_{\Lambda}^{\tau} f\right) .
$$

Here and in the sequel we let, for a probability measure $\mu, \mu(f)=\int f d \mu$, $\mu(f, g)=\mu((f-\mu(f))(g-\mu(g)))$, and $\operatorname{Ent}_{\mu}\left(f^{2}\right)=\mu\left(f^{2} \log f^{2}\right)-\mu\left(f^{2}\right)$ $\times \log \mu\left(f^{2}\right)$. The spectral gap controls the convergence to equilibrium in $L^{2}\left(\mu_{\Lambda}^{\tau}\right)$, while the logarithmic-Sobolev constant is related to hypercontractivity of the semigroup $\exp \left(t \mathcal{L}_{\Lambda}^{\tau}\right)$ (see [7]), and may be used to give bounds on the decay of the relative entropy of the law of the system at time $t$ with respect to $\mu_{\Lambda}^{\tau}$ as well as the total variation distance to $\mu_{\Lambda}^{\tau}$ (see, e.g., [9]). It is known [9] that $\operatorname{gap}\left(\mathcal{L}_{\Lambda}^{\tau}\right) \geq 2 S\left(\mathcal{L}_{\Lambda}^{\tau}\right)$, so that a uniform lower bound for $S\left(\mathcal{L}_{\Lambda}^{\tau}\right)$ implies a uniform lower bound for gap $\left(\mathcal{L}_{\Lambda}^{\tau}\right)$. For systems with finite or compact spin space, it is known that, for $\beta$ small enough, $S\left(\mathcal{L}_{\Lambda}^{\tau}\right)$ [and, henceforth, $\operatorname{gap}\left(\mathcal{L}_{\Lambda}^{\tau}\right)$ ] is uniformly bounded away from zero.

In the case of $\mathbf{Z}_{+}$-valued spins the picture changes drastically, since positivity of the spectral gap and of the logarithmic-Sobolev constant is nontrivial even for $\beta=0$ (infinite temperature, or no interaction). In absence of interaction, the dynamics are those of a family of independent identically distributed Markov processes, one for each site of $\Lambda$, all having $\rho$ as reversible measure (reference dynamics). We call $\mathcal{L}_{0}$ the generator of this process; we choose, but other choices would lead to the same results, $\mathscr{L}_{0}=-\nabla^{*} \nabla$, where, for $f: \mathbf{Z}_{+} \rightarrow \mathbf{R}, \nabla f(n)=$ $f(n+1)-f(n)$, and $\nabla^{*}$ is the adjoint of $\nabla$ in $L^{2}(\rho)$. It is well known that the spectral gap and the logarithmic-Sobolev constant for this system of i.i.d. processes equal the corresponding constants for a single component. Thus, for the noninteracting case, we are led to study the inequalities

$$
\rho(f, f) \leq l^{-1} \rho\left[(\nabla f)^{2}\right]
$$

and, for $f>0$,

$$
\operatorname{Ent}_{\rho}(f) \leq \alpha^{-1} \rho\left[(\nabla \sqrt{f})^{2}\right] .
$$

Both inequalities (1.1) and (1.2) are well understood in the sense that necessary and sufficient conditions on a probability $\rho$ on $\mathbf{Z}_{+}$are known for the strict positivity of gap $\left(\mathscr{L}_{0}\right)$ and $S\left(\mathscr{L}_{0}\right)$ [11]. In particular, for geometric and Poisson measures, $\operatorname{gap}\left(\mathscr{L}_{0}\right)>0$ but $S\left(\mathcal{L}_{0}\right)=0$. In order to have $S\left(\mathscr{L}_{0}\right)>0$ we need a $\rho$ with smaller tails, for example, $\rho(n) \propto e^{-c n^{2}}, c>0$. At finite temperature $(\beta>0)$, we show that whenever $\operatorname{gap}\left(\mathscr{L}_{0}\right)>0$ we have $\inf _{\Lambda, \tau} \operatorname{gap}\left(\mathcal{L}_{\Lambda}^{\tau}\right)>0$, provided $\beta$ is small enough to imply a suitable mixing condition on $\mu_{\Lambda}^{\tau}$. Conversely, if gap $\left(\mathscr{L}_{0}\right)=0$ [resp. $\left.S\left(\mathcal{L}_{0}\right)=0\right]$ then $\operatorname{gap}\left(\mathcal{L}_{\Lambda}^{\tau}\right)=0$ [resp. $S\left(\mathcal{L}_{\Lambda}^{\tau}\right)=0$ ] for every $\Lambda, \tau$. 
The main motivation for this paper is to go beyond the spectral gap. We are interested, in particular, in the choice $\rho=$ Poisson measure. The resulting system may be thought of as a discrete version of the birth and death process in the continuum studied in [2], where a uniform lower bound on the spectral gap is given. However, the logarithmic-Sobolev inequality fails for this reference measure. To our knowledge, the first modified version of the logarithmic-Sobolev inequality that holds for Poisson measures was proposed by Bobkov and Ledoux in [3]:

$$
\operatorname{Ent}_{\rho}(f) \leq \gamma^{-1} \rho\left[\frac{(\nabla f)^{2}}{f}\right]
$$

for $f>0$. In this paper we consider the following inequality:

$$
\operatorname{Ent}_{\rho}(f) \leq s^{-1} \rho[\nabla f \nabla \log f]
$$

for $f>0$, that, for the interacting system, becomes

$$
\operatorname{Ent}_{\mu_{\Lambda}^{\tau}}(f) \leq-s^{-1} \mu_{\Lambda}^{\tau}\left[f \mathcal{L}_{\Lambda}^{\tau} \log f\right] .
$$

Inequalities (1.4) and (1.5) will be called entropy inequalities, and we denote by $s\left(\mathcal{L}_{0}\right)$ and $s\left(\mathcal{L}_{\Lambda}^{\tau}\right)$ the corresponding optimal constants. The entropy inequalities arise quite naturally since they are equivalent to the exponential decay of the relative entropy with respect to the reversible measure (see Section 2). Since (see, e.g., the proof of Theorem 3.6 in [5]) $\mu_{\Lambda}^{\tau}\left[f \mathcal{L}_{\Lambda}^{\tau} \log f\right] \leq 4 \mu_{\Lambda}^{\tau}\left[\sqrt{f} \mathcal{L}_{\Lambda}^{\tau} \sqrt{f}\right]$, it is easily seen that $4 s\left(\mathcal{L}_{\Lambda}^{\tau}\right) \geq S\left(\mathcal{L}_{\Lambda}^{\tau}\right)$, that expresses the widely used fact that the logarithmic-Sobolev inequality implies exponential decay of entropy. Moreover, a simple perturbation argument similar to the one in [5], Lemma 3.1, shows that $s\left(\mathcal{L}_{\Lambda}^{\tau}\right) \leq 2 \operatorname{gap}\left(\mathcal{L}_{\Lambda}^{\tau}\right)$. Thus the entropy inequality is intermediate between the Poincaré inequality and the logarithmic-Sobolev inequality (for more generalities of these inequalities see [1]). As the main result of this paper, we show that $\inf _{\Lambda, \tau} s\left(\mathscr{L}_{\Lambda}^{\tau}\right)>0$ whenever $s\left(\mathscr{L}_{0}\right)>0$ and $\beta$ is sufficiently small, and $s\left(\mathscr{L}_{0}\right)>0$ for Poisson measures $\rho$. Moreover, we show that whenever one of the inequalities (1.1), (1.2) or (1.4) holds for the reference dynamics then, for $\beta$ small enough, the corresponding inequality for the interacting system holds uniformly in $\Lambda, \tau$. Unlike for the spectral gap and the logarithmic-Sobolev constant, we are not able to characterize those measures $\rho$ for which $s\left(\mathscr{L}_{0}\right)>0$. A sufficient condition is given in [12]: this condition is very hard to check, and, to our knowledge, has not been shown to be satisfied by any nontrivial measure, not even Poisson measures. In our view, providing better conditions is a very interesting open problem.

In the proof of the lower bound for $s\left(\mathcal{L}_{\Lambda}^{\tau}\right)$ we follow, as a guiding strategy, the recursive argument in [10]. The (nontrivial) adaptation of that argument to the entropy inequality had led us to a different proof, that is actually simpler than the original one in [10]. Our proof is based on a simple inequality that expresses "quasi" additivity of the entropy in disjoint volumes. After our proof was completed, we have been informed that a similar inequality has been independently obtained by Cesi [4]. 


\section{Notation and results.}

The Gibbs measures. For $\Lambda \subset \mathbf{Z}^{d}$ we denote by $|\Lambda|$ the cardinality of $\Lambda$, and if $|\Lambda|<+\infty$ we write $\Lambda \subset \subset \mathbf{Z}^{d}$. We consider the configuration space $\Omega=\mathbf{Z}_{+}^{\mathbf{Z}^{d}}$. An element $\eta \in \Omega$ is a function from $\mathbf{Z}^{d}$ in $\mathbf{Z}_{+}$; we denote by $\eta_{x}$ its value at $x \in \mathbf{Z}^{d}$. We endow $\Omega$ with the Borel $\sigma$-field $\mathcal{F}$ generated by the open sets of the product topology. If $\Lambda \subset \mathbf{Z}^{d}$, we denote by $\Omega_{\Lambda}:=\mathbf{Z}_{+}^{\Lambda}$ the reduced configuration space and by $\mathcal{F}_{\Lambda}$ the $\sigma$-field generated by $\left\{\eta_{x}: x \in \Lambda\right\}$. If $f$ is a function defined on $\Omega$ we write $f \in \mathcal{F}_{\Lambda}$ to indicate that $f$ is $\mathcal{F}_{\Lambda}$-measurable. Given $\eta \in \Omega$ and $\Lambda \subset \mathbf{Z}^{d}$ we denote with $\eta_{\Lambda}$ the restriction of $\eta$ on $\Omega_{\Lambda}$. For $A, B \subset \mathbf{Z}^{d}, \eta \in \Omega_{A}$ and $\omega \in \Omega_{B}$ we define $\eta \omega \in \Omega_{A \Delta B}$, where $A \Delta B:=(A \backslash B) \cup(B \backslash A)$ denotes the symmetric difference between $A$ and $B$, as

$$
(\eta \omega)_{x}:= \begin{cases}\eta_{x}, & x \in A \backslash B, \\ \omega_{x}, & x \in B \backslash A .\end{cases}
$$

For $x \in \mathbf{Z}^{d}$ we denote by $|x|:=\max _{k=1, \ldots, d}\left|x_{k}\right|$, and, for $A, B \subset \mathbf{Z}^{d}$, the distance beetwen $A$ and $B$ is denoted by

$$
\operatorname{dist}(A, B):=\min \{|x-y|: x \in A, y \in B\} .
$$

Let $\Phi:=\left\{\Phi_{R}: R \subset \mathbf{Z}^{d}\right\}$ be a bounded finite range potential, with range $r>0$, that is, for every $R \subset \mathbf{Z}^{d}, \Phi_{R}$ is a function $\Phi_{R}: \Omega_{R} \rightarrow \mathbf{R}$ such that

$$
\|\Phi\|:=\sup _{x \in \mathbf{Z}^{d}} \sum_{R \ni x}\left\|\Phi_{R}\right\|_{+\infty}<+\infty
$$

and $\Phi_{R} \equiv 0$ if $\operatorname{diam}(R)>r$. Given the potential $\left\{\Phi_{R}: R \subset \mathbf{Z}^{d}\right\}$ and $\Lambda \subset \subset \Omega$, the Hamiltonian $H_{\Lambda} \equiv H_{\Lambda, \Phi}$ is defined by

$$
H_{\Lambda}(\eta):=\sum_{R \cap \Lambda \neq \varnothing} \Phi_{R}\left(\eta_{R}\right) .
$$

For $\eta, \tau \in \Omega$ we also set $H_{\Lambda}^{\tau}(\eta):=H_{\Lambda}\left(\eta_{\Lambda} \tau_{\Lambda^{c}}\right) ; \tau$ in this case is called the boundary condition.

The reference probability for a single spin $n \in \mathbf{Z}_{+}$will be denoted by $\rho(\cdot)$ and it will be taken to be either a Poisson measure

$$
\rho(n)=e^{-\lambda} \frac{\lambda^{n}}{n !}
$$

for some $\lambda>0$, or a geometric measure

$$
\rho(n)=(1-c) c^{n}
$$

for some $0<c<1$. From now on the constants $\lambda$ and $c$ will be kept fixed, and dependence of other constants on $\lambda$ and $c$ will be omitted. 
For $\Lambda \subset \subset \mathbf{Z}^{d}, \tau \in \Omega$ and $\beta>0$ the finite volume Gibbs measure in $\Lambda$ at inverse temperature $\beta$ and boundary condition $\tau$ is the probability measure on $\left(\Omega_{\Lambda}, \mathcal{F}_{\Lambda}\right)$ given by

$$
\mu_{\Lambda}^{\tau}(\eta):=\left(Z_{\Lambda}^{\tau}(\beta)\right)^{-1} \exp \left[-\beta H_{\Lambda}^{\tau}(\eta)\right] \prod_{x \in \Lambda} \rho\left(\eta_{x}\right),
$$

where $Z_{\Lambda}^{\tau}(\beta)$, the partition function, is the appropriate normalization factor. For $f, g \in \mathcal{F}$ we denote with $\mu_{\Lambda}^{\tau}(f)$ the expectation of $f$ w.r.t. $\mu_{\Lambda}^{\tau}$, and with $\mu_{\Lambda}^{\tau}(f, g)=\mu_{\Lambda}^{\tau}(f g)-\mu_{\Lambda}^{\tau}(f) \mu_{\Lambda}^{\tau}(g)$ the covariance w.r.t. $\mu_{\Lambda}^{\tau}$. For $A \subset \Lambda$, we denote with $\mu_{\Lambda, A}$ the marginal of $\mu_{\Lambda}$ in $\Omega_{A}$, that is, $\mu_{\Lambda}(f)=\mu_{\Lambda, A}(f)$ for any $f$ $\mathcal{F}_{A}$-measurable. Given two probability measures $\mu$ and $v$ on $\left(\Omega_{\Lambda}, \mathcal{F}_{\Lambda}\right)$, we denote the relative entropy of $v$ w.r.t. $\mu$ by

$$
\mathbf{H}(v \mid \mu):= \begin{cases}\mu(f \log f), & \text { if } d v=f d \mu, f \log f \in L^{1}(\mu), \\ +\infty, & \text { if } v \ll \mu\end{cases}
$$

and the total variation distance between $\mu$ and $v$ by

$$
\|\mu-v\|_{\mathrm{TV}}=\frac{1}{2} \sum_{\eta \in \Omega_{\Lambda}}|\mu(\eta)-v(\eta)|=\max _{X \subset \Omega_{\Lambda}}|\mu(X)-v(X)| .
$$

Finally, for $f>0$, we let

$$
\operatorname{Ent}_{\mu}(f)=\mu(f \log f)-\mu(f) \log \mu(f)
$$

for $f \log f \in L^{1}(\mu)$, and $\operatorname{Ent}_{\mu}(f)=+\infty$ otherwise. It is known that $\operatorname{Ent}_{\mu}(f)=0$ if and only if $f$ is constant $\mu$-a.s. Note finally that $\mathbf{H}(\nu \mid \mu)=\operatorname{Ent}_{\mu}\left(\frac{d v}{d \mu}\right)$.

The dynamics. For a given function $f$ on $\Omega$ we let

$$
\begin{aligned}
& \left(\nabla_{x}^{-} f\right)(\eta):=\mathbf{1}_{\left\{\eta_{x}>0\right\}}\left[f\left(\eta-\delta^{x}\right)-f(\eta)\right], \\
& \left(\nabla_{x}^{+} f\right)(\eta):=f\left(\eta+\delta^{x}\right)-f(\eta), \quad x \in \mathbf{Z}^{d},
\end{aligned}
$$

where $\delta^{x} \in \Omega,\left(\delta^{x}\right)_{x}=1,\left(\delta^{x}\right)_{y}=0$ for any $y \neq x$. The stochastic dynamics we want to study are determined by the Markov generator $\mathcal{L}_{\Lambda}^{\tau}, \Lambda \subset \subset \mathbf{Z}^{d}, \tau \in \Omega$, defined by

$$
\left(\mathcal{L}_{\Lambda}^{\tau} f\right)(\eta):=\sum_{\substack{x \in \Lambda \\ * \in\{-,+\}}} c_{\Lambda}^{\tau}(x, \eta, *)\left(\nabla_{x}^{*} f\right)(\eta) .
$$

We require that the rates $c_{\Lambda}^{\tau}(\cdot, \cdot, \cdot)$ satisfy the detailed balance condition w.r.t. $\mu_{\Lambda}^{\tau}$ :

$$
c_{\Lambda}^{\tau}(x, \eta, \pm) \mu_{\Lambda}^{\tau}(\eta)=c_{\Lambda}^{\tau}\left(x, \eta \pm \delta_{x}, \mp\right) \mu_{\Lambda}^{\tau}\left(\eta \pm \delta_{x}\right) \quad \forall \eta \in \Omega_{\Lambda}, \forall x \in \Lambda,
$$

that is, $\mathcal{L}_{\Lambda}^{\tau}$ is self-adjoint in $L^{2}\left(\mu_{\Lambda}^{\tau}\right)$. (Note: the identities involving $\eta-\delta_{x}$ hold whenever $\eta_{x}>0$.) Moreover, we assume that there exists a constant $C>0$ dependent only on $\beta$ and $\|\Phi\|$, such that

$$
C^{-1} \bar{c}(x, \eta, \pm) \leq c_{\Lambda}^{\tau}(x, \eta, \pm) \leq C \bar{c}(x, \eta, \pm) \quad \forall \eta, \tau \in \Omega_{\Lambda}, x \in \mathbf{Z}^{d},
$$


where $\bar{c}(x, \eta, \pm)$ are the rates for a system with no interaction, that will be chosen as follows:

$$
\bar{c}(x, \eta,+)=1, \quad \bar{c}(x, \eta,-)=\frac{\eta_{x}}{\lambda},
$$

when $\rho$ is a Poisson measure of parameter $\lambda$, and

$$
\bar{c}(x, \eta,+)=1, \quad \bar{c}(x, \eta,-)=\frac{1}{c},
$$

when $\rho$ is a geometric measure of parameter $c$. An example of rates satisfying these conditions is

$$
c_{\Lambda}^{\tau}(x, \eta, \pm):=\bar{c}(x, \eta, \pm) \exp \left[-\frac{\beta}{2} \nabla_{x}^{ \pm} H_{\Lambda}^{\tau}(\eta)\right] .
$$

The Dirichlet form associated with $\mathcal{L}_{\Lambda}^{\tau}$ is given by

$$
\mathcal{E}_{\Lambda}^{\tau}(f, g):=-\mu_{\Lambda}^{\tau}\left(f \mathcal{L}_{\Lambda}^{\tau} g\right)=\sum_{x \in \Lambda} \mu_{\Lambda}^{\tau}\left(c_{\Lambda}^{\tau}(x, \cdot,+)\left(\nabla_{x}^{+} f\right)(\cdot)\left(\nabla_{x}^{+} g\right)(\cdot)\right),
$$

and we denote by $\left\{e^{t \mathcal{L}_{\Lambda}^{\tau}}\right\}_{t \geq 0}$ the Markov semigroup generated by $\mathcal{L}_{\Lambda}^{\tau}$ in $L^{2}\left(\mu_{\Lambda}^{\tau}\right)$.

The spectral gap, $\operatorname{gap}\left(\mathcal{L}_{\Lambda}^{\tau}\right)$, is defined by

$$
\operatorname{gap}\left(\mathcal{L}_{\Lambda}^{\tau}\right):=\inf \left\{\frac{\mathcal{E}_{\Lambda}^{\tau}(f, f)}{\mu_{\Lambda}^{\tau}(f, f)}, f \in L^{2}\left(\mu_{\Lambda}^{\tau}\right), \mu_{\Lambda}^{\tau}(f, f) \neq 0\right\} .
$$

It is well known that the following inequality, concerning convergence to equilibrium for the semigroup $\left\{e^{t \mathcal{L}_{\Lambda}^{\tau}}\right\}_{t \geq 0}$, holds:

$$
\left\|e^{t \mathcal{L}_{\Lambda}^{\tau}} f-\mu_{\Lambda}^{\tau}(f)\right\|_{L^{2}\left(\mu_{\Lambda}^{\tau}\right)} \leq\|f\|_{L^{2}\left(\mu_{\Lambda}^{\tau}\right)} e^{-g t / 2},
$$

where $g=\operatorname{gap}\left(\mathcal{L}_{\Lambda}^{\tau}\right)$.

The entropy constant $s\left(\mathcal{L}_{\Lambda}^{\tau}\right)$ is defined as

$$
s\left(\mathcal{L}_{\Lambda}^{\tau}\right):=\inf \left\{\frac{\mathcal{E}_{\Lambda}^{\tau}(f, \log f)}{\operatorname{Ent}_{\mu_{\Lambda}^{\tau}}(f)}: f \geq 0, f \log f \in L^{1}\left(\mu_{\Lambda}^{\tau}\right), \operatorname{Ent}_{\mu_{\Lambda}^{\tau}}(f) \neq 0\right\}
$$

or, equivalently, as the optimal constant for the entropy inequality

$$
\operatorname{Ent}_{\mu_{\Lambda}^{\tau}}(f) \leq s^{-1} \mathcal{E}_{\Lambda}^{\tau}(f, \log f) .
$$

The entropy inequality forces the relative entropy with respect to the invariant measure to decay exponentially (see, e.g., [13]):

$$
\mathbf{H}\left(v_{t} \mid \mu_{\Lambda}^{\tau}\right) \leq e^{-s t} \mathbf{H}\left(v \mid \mu_{\Lambda}^{\tau}\right),
$$

where $v_{t}:=v e^{t \mathcal{L}_{\Lambda}^{\tau}}$ is the distribution at time $t$ of the system having $v$ as initial distribution. In particular, by using the Csizar's inequality,

$$
\|\mu-v\|_{\mathrm{TV}} \leq \sqrt{\frac{1}{2} \mathbf{H}(v \mid \mu)},
$$


we get

$$
\left\|v_{t}-\mu_{\Lambda}^{\tau}\right\|_{\mathrm{TV}} \leq \sqrt{\frac{1}{2} \mathbf{H}\left(\nu \mid \mu_{\Lambda}^{\tau}\right)} e^{-s t / 2} .
$$

Finally, the logarithmic-Sobolev constant $S\left(\mathcal{L}_{\Lambda}^{\tau}\right)$ is defined as

$$
S\left(\mathcal{L}_{\Lambda}^{\tau}\right):=\inf \left\{\frac{\mathcal{E}_{\Lambda}^{\tau}(\sqrt{f}, \sqrt{f})}{\operatorname{Ent}_{\mu_{\Lambda}^{\tau}}(f)}: f \geq 0, f \log f \in L^{1}\left(\mu_{\Lambda}^{\tau}\right), \operatorname{Ent}_{\mu_{\Lambda}^{\tau}}(f) \neq 0\right\} .
$$

In order to state and prove our main result we need to assume a mixing property of the Gibbs measure. We introduce the family of fat rectangles of size smaller than $L$ (see [9]) in the following way. Let $R\left(x ; l_{1}, \ldots, l_{d}\right)$ denote the rectangle

$$
R\left(x ; l_{1}, \ldots, l_{d}\right)=x+\left(\left[1, l_{1}\right] \times \cdots \times\left[1, l_{d}\right]\right) \cap \mathbf{Z}^{d}, \quad x \in \mathbf{Z}^{d},\left(l_{1}, \ldots, l_{d}\right) \in \mathbf{Z}_{+}^{d} .
$$

The size of $R\left(x ; l_{1}, \ldots, l_{d}\right)$ is the number $\max \left\{l_{k}: k=1, \ldots, d\right\}$, and we say that $R\left(x ; l_{1}, \ldots, l_{d}\right)$ is fat if $\min \left\{l_{k}: k=1, \ldots, d\right\} \geq \frac{1}{10} \max \left\{l_{k}: k=1, \ldots, d\right\}$. Let $\mathcal{R}_{L}$ be the class of all fat rectangles in $\mathbf{Z}^{d}$ of size $L \in \mathbf{Z}_{+}$, and $\mathcal{R}=\bigcup_{L \geq 1} \mathcal{R}_{L}$.

Condition 2.1 (Mixing condition). Let $\Lambda \in \mathcal{R}$, for example, $\Lambda \in \mathcal{R}_{L}$ and $A, B \subset \Lambda$ such that $A, B \in \mathcal{R}_{L}$ and $A \cap B=\varnothing$. Then there exist constants $C_{1}(\beta, d,\|\Phi\|), C_{2}(\beta, d,\|\Phi\|)>0$ such that

$$
\sup _{\tau, \sigma \in \Omega}\left|\frac{\mu_{\Lambda}^{\tau}\left(\eta: \eta_{A}=\sigma_{A}\right) \mu_{\Lambda}^{\tau}\left(\eta: \eta_{B}=\sigma_{B}\right)}{\mu_{\Lambda}^{\tau}\left(\eta: \eta_{A \cup B}=\sigma_{A \cup B}\right)}-1\right| \leq C_{1} e^{-C_{2} \operatorname{dist}(A, B)} .
$$

It is standard to derive this condition from a condition on the exponential decay of covariances, see, for example, [9], Proposition 2.12, which can be derived by the "Dobrushin Condition" [6], which, in turn, holds true for $\beta$ sufficiently small.

We can now state our main results.

THEOREM 2.1. If Condition (2.1) holds true and the reference measure $\rho$ is either Poisson or geometric, then there exists a constant $\alpha>0$ independent of $\Lambda$ and $\tau$, such that

$$
\mu_{\Lambda}^{\tau}(f, f) \leq \alpha \mathcal{E}_{\Lambda}^{\tau}(f, f),
$$

for all $f \in L^{2}\left(\mu_{\Lambda}^{\tau}\right)$. In particular $\operatorname{gap}\left(\mathcal{L}_{\Lambda}^{\tau}\right) \geq \alpha^{-1}$.

THEOREM 2.2. If Condition (2.1), holds true and the reference measure is a Poisson measure, then there exists a constant $\alpha>0$ independent of $\Lambda$ and $\tau$, such that

$$
\operatorname{Ent}_{\mu_{\Lambda}^{\tau}}(f) \leq \alpha \mathcal{E}_{\Lambda}^{\tau}(f, \log f),
$$

for all $f \geq 0$ such that $f \log f \in L^{1}\left(\mu_{\Lambda}^{\tau}\right)$. In particular $s\left(\mathcal{L}_{\Lambda}^{\tau}\right) \geq \alpha^{-1}$. 
3. Single spin dynamics. In this section we analyze spectral gap and entropy constant for the noninteracting system with rates $\bar{c}(x, \eta, \pm)$. For such system, spins at different sites evolve through independent dynamics, whose infinitesimal generators are given by

$$
\mathcal{L}_{0} f(n)=[f(n+1)-f(n)]+\frac{n}{\lambda}[f(n-1)-f(n)]
$$

for $\rho=$ Poisson measure, and

$$
\mathscr{L}_{0} f(n)=[f(n+1)-f(n)]+\frac{\mathbf{1}_{n>0}}{c}[f(n-1)-f(n)]
$$

for $\rho=$ geometric measure. Note that in both (3.1) and (3.2) $\rho$ is a reversible measure for $\mathcal{L}_{0}$ [i.e., $\mathscr{L}_{0}$ is self-adjoint in $L^{2}(\rho)$ ], and the associated Dirichet form is given by

$$
\mathcal{E}_{0}(f, g)=-\sum_{n} \rho(n) f(n) \mathcal{L}_{0} f(n)=\sum_{n} \rho(n)[f(n+1)-f(n)]^{2} .
$$

First of all, for $\rho(n)=e^{-\lambda} \lambda^{n} / n$ !, we note that the logarithmic-Sobolev constant

$$
\inf \left\{\frac{\mathcal{E}_{0}(\sqrt{f}, \sqrt{f})}{\operatorname{Ent}_{\rho}(f)}: f \geq 0, f \log f \in L^{1}(\rho), \operatorname{Ent}_{\rho}(f) \neq 0\right\}
$$

vanishes, as shown by using the test functions $f_{N}=\mathbf{1}_{[N+1,+\infty)}$, and letting $N \uparrow+\infty$.

In the case of $\rho(n)=(1-c) c^{n}$, the entropy constant

$$
\inf \left\{\frac{\mathcal{E}_{0}(f, \log f)}{\operatorname{Ent}_{\rho}(f)}: f \geq 0, f \log f \in L^{1}(\rho), \operatorname{Ent}_{\rho}(f) \neq 0\right\}
$$

is shown to be zero by using the test functions $f_{a}(n)=a^{n}$, and letting $a \uparrow \frac{1}{c}$. Because of the general inequality $\varepsilon_{0}(f, \log f) \geq 4 \varepsilon_{0}(\sqrt{f}, \sqrt{f})$ (see, e.g., [5]), also the logarithmic-Sobolev constant is zero for geometric measures.

We now turn to positive results.

PROPOSITION 3.1. The entropy constant for a Poisson measure of mean $\lambda$ is greater or equal to $\lambda^{-1}$; that is, the inequality

$$
\operatorname{Ent}_{\rho}(f) \leq \lambda \varepsilon_{0}(f, \log f)
$$

holds for every $f>0$.

ProOF. We follow the scheme of the proof given in [3] for the inequality $\operatorname{Ent}_{\rho}(f) \leq \lambda \sum_{n} \rho(n) \frac{[f(n+1)-f(n)]^{2}}{f(n)}$. It is not clear to us whether there is any direct implication between this inequality and the entropy inequality.

The proof is based on classical binomial approximation for Poisson measures. Let $\mu_{p}$ be the Bernoulli measure on $\mathbf{Z}_{2}=\mathbf{Z} / 2 \mathbf{Z}$, with $\mu_{p}(1)=p \in(0,1)$. For 
$f: \mathbf{Z}_{2} \rightarrow \mathbf{R}$, we let $D f(x)=f(x+1)-f(x)$. We first show that, for all $f>0$ and all $p \in(0,1)$,

$$
\operatorname{Ent}_{\mu_{p}}(f) \leq p(1-p) \mu_{p}[(D f)(D \log f)] .
$$

To show (3.3), it is enough to observe the identity

$$
\operatorname{Ent}_{\mu_{p}}(f)=p(1-p) \mu_{p}[(D f)(D \log f)]+\mu_{p}(f) \mu_{p}\left[\log \left(\frac{f}{\mu_{p}(f)}\right)\right]
$$

and that, by Jensen's inequality, $\mu_{p}\left[\log \left(\frac{f}{\mu_{p}(f)}\right)\right] \leq 0$.

The rest of the proof is based on tensorization on (3.3), mimicking the argument in [3]. For $n>1$, let $\mu_{p}^{n}=\mu_{p} \otimes \cdots \otimes \mu_{p}$. For $f: \mathbf{Z}_{2}^{n} \rightarrow \mathbf{R}^{+}$we let $D_{i} f$ denote its discrete derivative w.r.t. the $i$ th variable, and $\operatorname{Ent}_{\mu_{p}}\left(f_{i}\right)$ denote the entropy of $f$ seen as a function of the $i$ th variable only, all others being frozen. The following inequality is a general property of entropy for product measures:

$$
\operatorname{Ent}_{\mu_{p}^{n}}(f) \leq \sum_{i=1}^{n} \int \operatorname{Ent}_{\mu_{p}}\left(f_{i}\right) d \mu_{p}^{n}
$$

Therefore one gets from (3.3),

$$
\operatorname{Ent}_{\mu_{p}^{n}}(f) \leq p(1-p) \sum_{i=1}^{n} E_{\mu_{p}^{n}}\left[\left(D_{i} f\right)\left(D_{i} \log f\right)\right]
$$

for all $f: \mathbf{Z}_{2}^{n} \rightarrow \mathbf{R}^{+}$. Now, take $f$ of the form $f\left(x_{1}, \ldots, x_{n}\right)=\varphi\left(x_{1}+x_{2}+\cdots+\right.$ $\left.x_{n}\right) \equiv \varphi\left(S_{n}\right)$, where $\varphi: \mathbf{Z}_{+} \rightarrow \mathbf{R}^{+}$is such that $\log \varphi$ is bounded. Here, the sum $x_{1}+x_{2}+\cdots+x_{n}$ in the argument of $\varphi$ is intended as a sum in $\mathbf{Z}$, not in $\mathbf{Z}_{2}$. Note that

$$
\begin{aligned}
\sum_{i=1}^{n}\left(D_{i} f\right)\left(D_{i} \log f\right)\left(x_{1}, \ldots, x_{n}\right) \\
=\left(n-S_{n}\right)\left[\varphi\left(S_{n+1}\right)-\varphi\left(S_{n}\right)\right]\left[\log \varphi\left(S_{n+1}\right)-\log \varphi\left(S_{n}\right)\right] \\
\quad+S_{n}\left[\varphi\left(S_{n-1}\right)-\varphi\left(S_{n}\right)\right]\left[\log \varphi\left(S_{n-1}\right)-\log \varphi\left(S_{n}\right)\right] .
\end{aligned}
$$

Thus, letting $p=\lambda / n$ in (3.6), we get

$$
\begin{aligned}
& \operatorname{Ent}_{\mu_{\lambda / n}^{n}}\left(\varphi\left(S_{n}\right)\right) \\
& \begin{aligned}
\text { (3.7) } \leq \frac{\lambda}{n}\left(1-\frac{\lambda}{n}\right) \mu_{\lambda / n}^{n}[(n & \left.-S_{n}\right)\left[\varphi\left(S_{n+1}\right)-\varphi\left(S_{n}\right)\right]\left[\log \varphi\left(S_{n+1}\right)-\log \varphi\left(S_{n}\right)\right] \\
& \left.+S_{n}\left[\varphi\left(S_{n-1}\right)-\varphi\left(S_{n}\right)\right]\left[\log \varphi\left(S_{n-1}\right)-\log \varphi\left(S_{n}\right)\right]\right] .
\end{aligned}
\end{aligned}
$$

By binomial approximation for Poisson distribution, the law of $S_{n}$ under $\mu_{\lambda / n}^{n}$ converges to $\rho$, and $\frac{1}{n} \mu_{\lambda / n}^{n}\left(S_{n}\right) \rightarrow 0$. Therefore, by letting $n \rightarrow \infty$ in (3.7), one obtains the desired entropy inequality for $\varphi>0$ with $\log \varphi$ bounded. An elementary approximation procedure extends it to all $\varphi>0$ with $\varphi \log \varphi \in L^{1}(\rho)$. 
PROPOSITION 3.2. For $\rho(n)=(1-c) c^{n}$, the generator $\mathscr{L}_{0}$ has a positive spectral gap.

The result follows from [8], Corollary 5.5, or from the general criterion in [11]. After having shown positivity of the entropy constant and of the spectral gap for the reference dynamics of a single spin, we now show the basic fact that, for a fixed $\Lambda$, the corresponding constants for $\mathcal{L}_{\Lambda}^{\tau}$ are strictly positive, although possibly depending on $\Lambda$, if and only if they are strictly positive for the reference dynamics. This is a basic and general fact, that does not depend on the Mixing Condition 2.1, but only on the boundedness of the potential.

PROPOSITION 3.3. Let $k(\mathcal{L})$ be either one of $\operatorname{gap}(\mathcal{L}), s(\mathcal{L})$ or $S(\mathcal{L})$ associated to a generator $\mathcal{L}$. For every $\Lambda \subset \subset \mathbf{Z}^{d}$, there is a constant $A>0$, possibly depending on $|\Lambda|$ but not on $\tau \in \Omega$, such that

$$
A^{-1} k\left(\mathcal{L}_{0}\right) \leq k\left(\mathcal{L}_{\Lambda}^{\tau}\right) \leq A k\left(\mathcal{L}_{0}\right) .
$$

PROOF. For the given $\Lambda$, we consider a system in $\Omega_{\Lambda}$ whose spins evolve independently with dynamics generated by $\mathcal{L}_{0}$. The infinitesimal generator of this process is

$$
\mathcal{L}_{0, \Lambda} f(\eta):=\sum_{x \in \Lambda} \mathcal{L}_{x} f(\eta),
$$

where $\mathcal{L}_{x}$ denotes the operator $\mathcal{L}_{0}$ acting on the spin $\eta_{x}$. The homogeneous product measure $\rho_{\Lambda}:=\rho^{\otimes \Lambda}$ is reversible for $\mathcal{L}_{0, \Lambda}$. We denote by $\varepsilon_{0, \Lambda}$ the corresponding Dirichlet form. As in the proof of Proposition 3.1 [see (3.5)], the behavior of the entropy under tensorization, guarantees that $s\left(\mathscr{L}_{0, \Lambda}\right)=$ $s\left(\mathscr{L}_{0}\right)$. Similarly (see, e.g., [5]), one shows that gap $\left(\mathscr{L}_{0, \Lambda}\right)=\operatorname{gap}\left(\mathscr{L}_{0}\right)$. Moreover, trivially, $S\left(\mathscr{L}_{0, \Lambda}\right)=0$. Thus, to get (3.8), it is enough to show that

$$
\begin{aligned}
B^{-1} \mathcal{E}_{0, \Lambda}(f, f) & \leq \mathcal{E}_{\Lambda}^{\tau}(f, f) \leq B \mathcal{E}_{0, \Lambda}(f, f), \\
B^{-1} \mathcal{E}_{0, \Lambda}(f, \log f) & \leq \mathcal{E}_{\Lambda}^{\tau}(f, \log f) \leq B \mathcal{E}_{0, \Lambda}(f, \log f), \\
B^{-1} \rho_{\Lambda}(f, f) & \leq \mu_{\Lambda}^{\tau}(f, f) \leq B \rho_{\Lambda}(f, f), \\
B^{-1} \operatorname{Ent}_{\rho_{\Lambda}}(f) & \leq \operatorname{Ent}_{\mu_{\Lambda}^{\tau}}(f) \leq B \operatorname{Ent}_{\rho_{\Lambda}}(f),
\end{aligned}
$$

for every $f$ [positive in (3.10) and (3.12)], with the constant $B$ depending on $|\Lambda|$ but not on $\tau$. Inequalities (3.9) and (3.10) are simple consequences of (2.2), after having noticed that $\bar{c}(x, \eta, \pm)$ are just the rates corresponding to $\mathcal{L}_{0, \Lambda}$. Inequality (3.11) is easily derived from the uniform estimate

$$
e^{-2|\Lambda| \beta\|\Phi\|} \leq \frac{d \mu_{\Lambda}^{\tau}}{d \rho_{\Lambda}} \leq e^{2|\Lambda| \beta\|\Phi\|} .
$$

Finally, inequality (3.12) comes from (3.13) as in Lemma 3.5 in [14]. 
4. Spectral gap. This section is devoted to the proof of Theorem 2.1. We begin by stating two lemmas concerning conditional variance. These lemmas would simplify the existing proofs of uniform positivity of the spectral gap even in the compact spin case.

In what follows, given a probability space $(\Omega, \mathcal{F}, \mu)$ and a $\sigma$-field $g \subset \mathcal{F}$, we let $\mu(f, g \mid g)$ be the conditional covariance of the random variables $f$ and $g$; that is,

$$
\mu(f, g \mid \mathcal{g})=\mu(f g \mid \mathcal{g})-\mu(f \mid \mathcal{g}) \mu(g \mid \mathcal{g})
$$

LEMMA 4.1. Let $(\Omega, \mathcal{F}, \mu)$ be a probability space, $\mathcal{F}_{1}, \mathcal{F}_{2}$ be sub- $\sigma$-fields of $\mathcal{F}$. Then the following inequality holds for every $f \in L^{2}(\mu)$ :

$$
\mu(f, f) \leq \mu\left(\mu\left(f, f \mid \mathcal{F}_{1}\right)+\mu\left(f, f \mid \mathcal{F}_{2}\right)\right)+2 \mu\left(\mu\left(f \mid \mathcal{F}_{1}\right), \mu\left(f \mid \mathcal{F}_{2}\right)\right) .
$$

PROOF. It is enough to prove (4.1) for $f$ such that $\mu(f)=0$. In this case (4.1) is equivalent to

$$
\mu\left(f^{2}\right)-\mu\left(\mu\left(f \mid \mathcal{F}_{1}\right)^{2}\right)-\mu\left(\mu\left(f \mid \mathcal{F}_{2}\right)^{2}\right)+2 \mu\left(\mu\left(f \mid \mathcal{F}_{1}\right) \mu\left(f \mid \mathcal{F}_{2}\right)\right) \geq 0 .
$$

Letting $P_{i} f=\mu\left(f \mid \mathcal{F}_{i}\right), i=1,2$, we have that $P_{i}$ is an orthogonal projection in the Hilbert space $L^{2}(\mu)$. If we denote by $\|\cdot\|$ and $\langle\cdot, \cdot\rangle$ the norm and the scalar product in $L^{2}(\mu)$, respectively, then (4.2) follows from the general inequality for orthogonal projection in Hilbert spaces,

$$
\|f\|^{2}-\left\|P_{1} f\right\|^{2}-\left\|P_{2} f\right\|^{2}+2\left\langle P_{1} f, P_{2} f\right\rangle \geq 0
$$

that is proved as follows:

$$
\begin{aligned}
\| P_{1} f & \left\|^{2}+\right\| P_{2} f \|^{2}-2\left\langle P_{1} f, P_{2} f\right\rangle \\
& =\left\|\left(P_{1}-P_{2}\right) f\right\|^{2} \\
& =\left\|\left[P_{1}\left(\mathbf{1}-P_{2}\right)-\left(\mathbf{1}-P_{1}\right) P_{2}\right] f\right\|^{2} \\
& =\left\|P_{1}\left(\mathbf{1}-P_{2}\right) f\right\|^{2}+\left\|\left(\mathbf{1}-P_{1}\right) P_{2} f\right\|^{2} \\
& \leq\left\|\left(\mathbf{1}-P_{2}\right) f\right\|^{2}+\left\|P_{2} f\right\|^{2}=\|f\|^{2} .
\end{aligned}
$$

The next elementary lemma, provides an estimate for the last summand of the right-hand side of (4.1).

LEMMA 4.2. With the notation of Lemma 4.1, suppose there exists a further probability measure $\bar{\mu}$ on $(\Omega, \mathcal{F})$ such that $\mathcal{F}_{1}$ and $\mathcal{F}_{2}$ are independent under $\bar{\mu}$, $\mu \ll \bar{\mu}$, and $\left.\mu\right|_{\mathcal{F}_{i}}=\left.\bar{\mu}\right|_{\mathcal{F}_{i}}, i=1,2$, where $\left.\mu\right|_{\mathcal{F}_{i}}$ denotes the restriction of $\mu$ to $\mathcal{F}_{i}$. Then, for every $f \in L^{2}(\mu)$,

$$
\mu\left(\mu\left(f \mid \mathscr{F}_{1}\right), \mu\left(f \mid \mathcal{F}_{2}\right)\right) \leq\|h-1\|_{\infty} \mu(f, f),
$$

where $h=\frac{d \mu}{d \bar{\mu}}$. 
ProOF. We may assume $\mu(f)=0$. We obtain

$$
\begin{aligned}
\mu(\mu & \left.\left(f \mid \mathcal{F}_{1}\right), \mu\left(f \mid \mathcal{F}_{2}\right)\right) \\
& =\bar{\mu}\left((h-1) \mu\left(f \mid \mathcal{F}_{1}\right) \mu\left(f \mid \mathcal{F}_{2}\right)\right)+\bar{\mu}\left(\mu\left(f \mid \mathcal{F}_{1}\right) \mu\left(f \mid \mathcal{F}_{2}\right)\right) \\
& \leq\|h-1\|_{\infty} \mu\left(\left|\mu\left(f \mid \mathcal{F}_{1}\right)\right|\right) \mu\left(\left|\mu\left(f \mid \mathcal{F}_{2}\right)\right|\right) \\
& \leq\|h-1\|_{\infty} \mu\left(f^{2}\right),
\end{aligned}
$$

where we have used the facts that $\bar{\mu}\left(\mu\left(f \mid \mathcal{F}_{1}\right) \mu\left(f \mid \mathcal{F}_{2}\right)\right)=0$ and $\mu\left(\left|\mu\left(f \mid \mathcal{F}_{i}\right)\right|\right) \leq$ $\sqrt{\mu\left(\mu\left(f \mid \mathcal{F}_{i}\right)^{2}\right)} \leq \sqrt{\mu\left(f^{2}\right)}$.

Now we want to use inequality (4.1) to prove the existence of a spectral gap independent of the volume $\Lambda$ and of the boundary condition $\tau$. This can be done by a recursive analysis similar to the one in [9]. Let

$$
g(L):=\inf _{R \in \mathcal{R}_{L}} \inf _{\tau \in \Omega} \operatorname{gap}\left(\mathcal{L}_{\mathrm{R}}^{\tau}\right)
$$

By Proposition 3.3, $g(L)>0$ for every $L>0$, for both geometric and Poisson reference measure. Theorem 2.1 is an immediate consequence of the following result.

Proposition 4.1. Assume the Mixing Condition 2.1. Then there exists a positive constant $k=k(\beta,\|\Phi\|)$ such that

$$
g(2 L) \geq\left(1-\frac{k}{\sqrt{L}}\right) g(L)
$$

for $L$ large enough. In particular, this implies $\inf _{L} g(L)>0$.

Proof. Let us consider a rectangle $R:=R\left(x ; l_{1}, \ldots, l_{d}\right) \in \mathcal{R}_{2 L}$. Inequality (4.4) follows if we show that

$$
\operatorname{gap}\left(\mathscr{L}_{R}^{\tau}\right) \geq\left(1-\frac{k}{\sqrt{L}}\right) g(L)
$$

for a constant $k$ independent of $R, L$ and $\tau$. Without loss of generality we can take $x=0, \underline{l}:=l_{1}, \bar{l}:=l_{d}$ and $L<l_{d} \leq 2 L$. We set $a=[\sqrt{L}]$, where $[\cdot]$ denotes the integer part. For every integer $1 \leq n \leq \frac{L}{10 a}$ we cover $R$ with the pair of rectangles,

$$
\begin{aligned}
& A_{n}=\left\{x \in R: \frac{l_{d}}{2}+(n-1) a<x_{d} \leq l_{d}\right\}, \\
& B_{n}=\left\{x \in R: 1 \leq x_{d} \leq \frac{l_{d}}{2}+n a\right\} .
\end{aligned}
$$


Note that, for $n$ fixed, $A_{n}$ and $B_{n}$ are two overlapping rectangles, and the shortest side of the overlap has length proportional to $\sqrt{L}$. In what follows we apply Lemmas 4.1 and 4.2 with

$$
\mu=\mu_{R}^{\tau}, \quad \mathcal{F}_{1}=\sigma\left\{\eta_{i}: i \in R \backslash A_{n}\right\}, \quad \mathcal{F}_{2}=\sigma\left\{\eta_{i}: i \in R \backslash B_{n}\right\}
$$

and

$$
\bar{\mu}(\eta)=\mu_{A_{n} \cap B_{n}}^{\eta \tau}\left(\eta_{A_{n} \cap B_{n}}\right) \mu_{R, R \backslash A_{n}}^{\tau}\left(\eta_{R \backslash A_{n}}\right) \mu_{R, R \backslash B_{n}}^{\tau}\left(\eta_{R \backslash B_{n}}\right) .
$$

This measure $\bar{\mu}$ is obtained from $\mu$ with a natural modification that makes $\mathcal{F}_{1}$ and $\widetilde{F}_{2}$ independent. Indeed, $\eta_{R \backslash A_{n}}$ and $\eta_{R \backslash B_{n}}$ are independent under $\bar{\mu}$ and have the same marginals as under $\mu$. Moreover, the distribution of $\eta_{A_{n} \cap B_{n}}$ conditioned to $\eta_{\left(A_{n} \cap B_{n}\right)^{c}}$ is the same under $\mu$ and under $\bar{\mu}$. Note that the assumptions of Lemma 4.2 are satisfied. Moreover, by Condition 2.1, for $h=\frac{d \mu}{d \bar{\mu}}$, we have

$$
\|h-1\|_{\infty} \leq e^{-C \sqrt{L}}
$$

for a suitable constant $C$ and $L$ large enough.

Now let us take a function $f$ with $\mu_{R}^{\tau}(f)=0$. By Lemma 4.1 we have

$$
\mu_{R}^{\tau}(f, f) \leq \mu_{R}^{\tau}\left(\mu_{A_{n}}^{\cdot}(f, f)+\mu_{B_{n}}^{\cdot}(f, f)\right)+2 \mu_{R}^{\tau}\left(\mu_{A_{n}}^{\cdot}(f) \mu_{B_{n}}^{\cdot}(f)\right),
$$

while by Lemma 4.2 and (4.5),

$$
\mu_{R}^{\tau}\left(\mu_{A_{n}}^{\cdot}(f) \mu_{B_{n}}^{\cdot}(f)\right) \leq e^{-C \sqrt{L}} \mu_{R}^{\tau}(f, f) .
$$

Moreover, by definition

$$
\mu_{A_{n}}^{\sigma}(f, f) \leq\left(\operatorname{gap}\left(\mathcal{L}_{A_{n}}^{\sigma}\right)\right)^{-1} \mathcal{E}_{A_{n}}^{\sigma}(f, f)
$$

and the same for the set $B_{n}$. So from (4.6) we obtain

$$
\begin{aligned}
\mu_{R}^{\tau}(f, f) \leq & {\left[\inf _{\sigma \in \Omega}\left(\operatorname{gap}\left(\mathcal{L}_{A_{n}}^{\sigma}\right) \wedge \operatorname{gap}\left(\mathcal{L}_{B_{n}}^{\sigma}\right)\right)\right]^{-1} \mu_{R}^{\tau}\left[\mathcal{E}_{\dot{A}_{n}}(f, f)+\mathcal{E}_{B_{n}}(f, f)\right] } \\
& +2 e^{-C \sqrt{L}} \mu_{R}^{\tau}(f, f)
\end{aligned}
$$

and so

$$
\mu_{R}^{\tau}(f, f) \leq \gamma\left(L, A_{n}, B_{n}\right)\left\{\mathcal{E}_{R}^{\tau}(f, f)+\mu_{R}^{\tau}\left(\sum_{x \in A_{n} \cap B_{n}} c_{\Lambda}^{\tau}(x, \cdot,+)\left|\nabla_{x}^{+} f\right|^{2}\right)\right\}
$$

where

$$
\gamma\left(L, A_{n}, B_{n}\right)=\frac{1}{1-2 e^{-C \sqrt{L}}}\left(\inf _{\sigma \in \Omega} \operatorname{gap}\left(\mathcal{L}_{A_{n}}^{\sigma}\right) \wedge \operatorname{gap}\left(\mathcal{L}_{B_{n}}^{\sigma}\right)\right)^{-1} .
$$

Now, let us observe that, as $n$ varies, the sets $A_{n} \cap B_{n}$ are disjoints, so that if we average over $n$ the equation (4.8), we obtain

$$
\mu_{R}^{\tau}(f, f) \leq\left(1+\left[\frac{L}{10 a}\right]^{-1}\right) \sup _{n} \gamma\left(L, A_{n}, B_{n}\right) \mathcal{E}_{R}^{\tau}(f, f) .
$$


It follows that

$$
\operatorname{gap}\left(\mathcal{L}_{R}^{\tau}\right) \geq\left(1-\frac{C}{\sqrt{L}}\right) \min _{n}\left(\inf _{\sigma \in \Omega} \operatorname{gap}\left(\mathcal{L}_{A_{n}}^{\sigma}\right) \wedge \operatorname{gap}\left(\mathcal{L}_{B_{n}}^{\sigma}\right)\right) .
$$

In order to derive (4.4) we have to analyze the spectral gap of the generator in the rectangles $A_{n}$ and $B_{n}$. Let us consider first the rectangle $B_{n}$ (the analysis is similar for $A_{n}$ ) and let us distinguish two different cases.

1. If $\max \left\{l_{k}: k=1, \ldots, d-1\right\} \leq \frac{3}{2} L$ then the longest side of $B_{n}$ is less or equal to $\frac{3}{2} L$, because also the side corresponding to the coordinate $x_{d}$, by the definition of $B_{n}$ is less than or equal to (1.2)L. In this case $B_{n} \in \mathcal{R}_{\frac{3}{2} L}$ and so $\operatorname{gap}\left(\mathcal{L}_{B_{n}}^{\tau}\right) \geq g\left(\frac{3}{2} L\right)$.

2. If there exists an index $i$ such that $l_{i}>\frac{3}{2} L$, then $B_{n} \in \mathcal{R}_{2 L}$, with the property that $\bar{l}:=l_{i}>\frac{3}{2} L$ and $\underline{l} \leq l_{d} \leq(1.2) L$. In this case

$$
\operatorname{gap}\left(\mathcal{L}_{B_{n}}^{\tau}\right) \geq \min _{\substack{R \in \mathcal{R}_{2 L} \\ \underline{l} \leq(1.2) L, \bar{l} \geq 3 / 2 L}} \operatorname{gap}\left(\mathcal{L}_{R}^{\tau}\right) .
$$

In conclusion we obtain that the right-hand side of (4.10) is larger than

$$
\left(1-\frac{C}{\sqrt{L}}\right)\left(g\left(\frac{3}{2} L\right) \wedge \min _{\substack{R \in \mathcal{R}_{2 L} \\ \underline{l} \leq 1.2 L, \bar{l} \geq 3 / 2 L}} \operatorname{gap}\left(\mathcal{L}_{R}^{\tau}\right)\right) .
$$

Now we consider a rectangle in the set $\mathcal{R}_{2 L}$ such that the shortest side is less than or equal to (1.2) $L$ and the longest one is greater than or equal to $\frac{3}{2} L$. Iterating the argument leading to (4.11) at most $d-1$ times on such rectangle, we end up with a rectangle whose longest side has length less than or equal to $\frac{3}{2} L$. Thus

$$
\min _{\substack{R \in \mathcal{R}_{2 L} \\ \underline{l} \leq(1.2) L, \bar{l} \geq 3 / 2 L}} \operatorname{gap}\left(\mathcal{L}_{R}^{\tau}\right) \geq\left(1-\frac{C}{\sqrt{L}}\right)^{d-1} g\left(\frac{3}{2} L\right) .
$$

By combining (4.11) and (4.12) we get

$$
g(2 L) \geq\left(1-\frac{C}{\sqrt{L}}\right)^{d} g\left(\frac{3}{2} L\right) .
$$

If we iterate two more times (4.13) we obtain

$$
g(2 L) \geq\left(1-\frac{C}{\sqrt{L}}\right)^{d}\left(1-\frac{C}{\sqrt{(3 / 4) L}}\right)^{d}\left(1-\frac{C}{\sqrt{(9 / 16) L}}\right)^{d} g(L),
$$

from which the conclusion follows. 
5. Entropy decay. This section is devoted to the proof of Theorem 2.2. The proof follows closely the proof of Theorem 2.1. In what follows, given a probability space $(\Omega, \mathcal{F}, \mu)$ and a $\sigma$-field $g \subset \mathcal{F}$, we define, for $f>0$, the conditional entropy $\operatorname{Ent}_{\mu}(f \mid \mathcal{g})$ as

$$
\operatorname{Ent}_{\mu}(f \mid \xi)=\mu(f \log f \mid \xi)-\mu(f \mid \mathcal{g}) \log \mu(f \mid \xi) .
$$

Similarly to Section 4, we state two lemmas concerning conditional entropy.

LEMMA 5.1. Let $(\Omega, \mathcal{F}, \mu)$ be a probability space, $\mathcal{F}_{1}, \mathcal{F}_{2}$ be sub- $\sigma$-fields of $\mathcal{F}$. Then the following inequality holds for every $f \geq 0$ such that $f \log f \in$ $L^{1}(\mu)$ and $\mu(f)=1$ :

$$
\operatorname{Ent}_{\mu}(f) \leq \mu\left[\operatorname{Ent}_{\mu}\left(f \mid \mathcal{F}_{1}\right)+\operatorname{Ent}_{\mu}\left(f \mid \mathcal{F}_{2}\right)\right]+\log \mu\left[\mu\left(f \mid \mathcal{F}_{1}\right) \mu\left(f \mid \mathcal{F}_{2}\right)\right] .
$$

PROOF. The right-hand side of (5.1) is equal to

$2 \mu(f \log f)-\mu\left[f \log \mu\left(f \mid \mathcal{F}_{1}\right)\right]-\mu\left[f \log \mu\left(f \mid \mathcal{F}_{2}\right)\right]+\log \mu\left[\mu\left(f \mid \mathcal{F}_{1}\right) \mu\left(f \mid \mathcal{F}_{2}\right)\right]$.

So (5.1) is equivalent to

$$
\mu(f \log f)-\mu\left[f \log \mu\left(f \mid \mathcal{F}_{1}\right) \mu\left(f \mid \mathcal{F}_{2}\right)\right]+\log \mu\left[\mu\left(f \mid \mathcal{F}_{1}\right) \mu\left(f \mid \mathcal{F}_{2}\right)\right] \geq 0 .
$$

This is trivially true because the left-hand side of (5.2) equals the relative entropy of $f d \mu$ with respect to $\frac{\mu\left(f \mid \mathcal{F}_{1}\right) \mu\left(f \mid \mathcal{F}_{2}\right)}{\mu\left[\mu\left(f \mid \mathcal{F}_{1}\right) \mu\left(f \mid \mathcal{F}_{2}\right)\right]} d \mu$.

LEMMA 5.2. With the same notation as in Lemma 5.1, suppose there exists a further probability measure $\bar{\mu}$ on $(\Omega, \mathcal{F})$ such that $\mathcal{F}_{1}$ and $\mathcal{F}_{2}$ are independent under $\bar{\mu}, \mu \ll \bar{\mu}$, and $\left.\mu\right|_{\mathscr{F}_{i}}=\left.\bar{\mu}\right|_{\mathcal{F}_{i}}, i=1,2$. Then, for every $f \geq 0$ such that $f \log f \in L^{1}(\mu)$ and $\mu(f)=1$,

$$
\left|\log \mu\left[\mu\left(f \mid \mathcal{F}_{1}\right) \mu\left(f \mid \mathcal{F}_{2}\right)\right]\right| \leq 4\|h-1\|_{\infty} \operatorname{Ent}_{\mu}(f),
$$

where $h=\frac{d \mu}{d \bar{\mu}}$.

Proof. Notice that

$$
\begin{aligned}
\log \mu & {\left[\mu\left(f \mid \mathcal{F}_{1}\right) \mu\left(f \mid \mathcal{F}_{2}\right)\right] } \\
& =\log \bar{\mu}\left[h \mu\left(f \mid \mathcal{F}_{1}\right) \mu\left(f \mid \mathcal{F}_{2}\right)\right] \\
& =\log \left\{\left[(h-1) \mu\left(f \mid \mathcal{F}_{1}\right) \mu\left(f \mid \mathcal{F}_{2}\right)\right]+\bar{\mu}\left[\mu\left(f \mid \mathcal{F}_{1}\right) \mu\left(f \mid \mathcal{F}_{2}\right)\right]\right\} \\
& =\log \left\{\bar{\mu}\left[(h-1) \mu\left(f \mid \mathcal{F}_{1}\right) \mu\left(f \mid \mathcal{F}_{2}\right)\right]+1\right\} \\
& \leq \bar{\mu}\left[(h-1) \mu\left(f \mid \mathcal{F}_{1}\right) \mu\left(f \mid \mathcal{F}_{2}\right)\right],
\end{aligned}
$$


where we use the elementary inequality $\log (x+1) \leq x$. This implies that

$$
\begin{aligned}
& \mid \log \mu[\left.\mu\left(f \mid \mathcal{F}_{1}\right) \mu\left(f \mid \mathcal{F}_{2}\right)\right] \mid \\
& \leq\left|\bar{\mu}\left[(h-1) \mu\left(f \mid \mathcal{F}_{1}\right) \mu\left(f \mid \mathcal{F}_{2}\right)\right]\right| \\
&=\mid \bar{\mu}\left\{(h-1)\left[\mu\left(f \mid \mathcal{F}_{1}\right)-\mu\left(\sqrt{\mu\left(f \mid \mathcal{F}_{1}\right)}\right)^{2}\right]\right. \\
&\left.\quad \times\left[\mu\left(f \mid \mathcal{F}_{2}\right)-\mu\left(\sqrt{\mu\left(f \mid \mathcal{F}_{2}\right)}\right)^{2}\right]\right\} \mid \\
& \leq\|h-1\|_{\infty} \bar{\mu}\left[\left|\mu\left(f \mid \mathcal{F}_{1}\right)-\mu\left(\sqrt{\mu\left(f \mid \mathcal{F}_{1}\right)}\right)^{2}\right|\right. \\
&\left.\left.\quad \times \mid \mu\left(f \mid \mathcal{F}_{2}\right)-\mu\left(\sqrt{\mu\left(f \mid \mathcal{F}_{2}\right.}\right)\right)^{2} \mid\right] \\
&=\|h-1\|_{\infty} \mu\left(\left|\mu\left(f \mid \mathcal{F}_{1}\right)-\mu\left(\sqrt{\mu\left(f \mid \mathcal{F}_{1}\right)}\right)^{2}\right|\right) \\
& \quad \times \mu\left(\left|\mu\left(f \mid \mathcal{F}_{2}\right)-\mu\left(\sqrt{\mu\left(f \mid \mathcal{F}_{2}\right)}\right)^{2}\right|\right) .
\end{aligned}
$$

Now notice that

$$
\mu\left(\left|\mu\left(f \mid \mathcal{F}_{k}\right)-\mu\left(\sqrt{\mu\left(f \mid \mathcal{F}_{k}\right)}\right)^{2}\right|\right) \leq 2 \sqrt{\mu(\sqrt{f}, \sqrt{f})}, \quad k=1,2 .
$$

In fact

$$
\begin{aligned}
& \mu\left(\left|\mu\left(f \mid \mathcal{F}_{k}\right)-\mu\left(\sqrt{\mu\left(f \mid \mathcal{F}_{k}\right)}\right)^{2}\right|\right) \\
& \quad=\mu\left[\left|\sqrt{\mu\left(f \mid \mathcal{F}_{k}\right)}-\mu\left(\sqrt{\mu\left(f \mid \mathcal{F}_{k}\right)}\right)\right|\left|\sqrt{\mu\left(f \mid \mathcal{F}_{k}\right)}+\mu\left(\sqrt{\mu\left(f \mid \mathcal{F}_{k}\right)}\right)\right|\right] \\
& \quad \leq \sqrt{\mu\left(\sqrt{\mu\left(f \mid \mathcal{F}_{k}\right)}, \sqrt{\mu\left(f \mid \mathcal{F}_{k}\right)}\right)} \sqrt{2\left[1+\mu\left(\sqrt{\mu\left(f \mid \mathcal{F}_{k}\right)}\right)^{2}\right]} \\
& \quad \leq 2 \sqrt{\mu\left(\sqrt{\mu\left(f \mid \mathcal{F}_{k}\right)}, \sqrt{\mu\left(f \mid \mathcal{F}_{k}\right)}\right)} \leq 2 \sqrt{\mu(\sqrt{f}, \sqrt{f})} .
\end{aligned}
$$

By (5.5) and (5.6) we get

$$
\left|\log \mu\left[\mu\left(f \mid \mathcal{F}_{1}\right) \mu\left(f \mid \mathcal{F}_{2}\right)\right]\right| \leq 4\|h-1\|_{\infty} \mu(\sqrt{f}, \sqrt{f}),
$$

and (5.3) follows because

$$
\mu(\sqrt{f}, \sqrt{f}) \leq \mu(f \log f)
$$

for every $f>0$ such that $\mu(f)=1$, see [4].

We can now prove Theorem 2.2. Because the proof follows closely the proof of Theorem 2.1 we give only a sketch. Define

$$
s(L):=\inf _{R \in \mathcal{R}_{L}^{d}} \inf _{\tau \in \Omega} s\left(\mathcal{L}_{R}^{\tau}\right) .
$$

By Proposition 3.3, $s(L)>0$ for every $L$, for the Poisson reference measure. Similarly to Theorem 2.1, Theorem 2.2 follows readily from the following proposition. 
Proposition 5.1. Assume Mixing Condition 2.1. Then there exists a positive constant $k=k(\beta,\|\Phi\|)$ such that

$$
s(2 L) \geq\left(1-\frac{k}{\sqrt{L}}\right) s(L)
$$

for L large enough. In particular, this implies $\inf _{L} s(L)>0$.

PROOF. With the same notation as Proposition 4.1 let $f$ be a nonnegative function such that $\mu_{R}^{\tau}(f)=1$ and $f \log f \in L^{1}\left(\mu_{R}^{\tau}\right)$. By (5.1),

$$
\begin{aligned}
\mu_{R}^{\tau}(f \log f) \leq & \mu_{R}^{\tau}\left[\mu_{A_{n}}\left(f \log \frac{f}{\mu_{A_{n}}(f)}\right)+\mu_{B_{n}}\left(f \log \frac{f}{\mu_{B_{n}}^{\cdot}(f)}\right)\right] \\
& +\log \mu_{R}^{\tau}\left[\mu_{A_{n}}^{\cdot}(f) \mu_{B_{n}}(f)\right],
\end{aligned}
$$

while by Lemma 5.2 and Condition 2.1,

$$
\left|\log \mu_{R}^{\tau}\left(\mu_{\dot{A}_{n}}(f) \mu_{B_{n}}(f)\right)\right| \leq 2 e^{-C \sqrt{L}} \mu_{R}^{\tau}(f \log f) .
$$

Moreover, by definition,

$$
\mu_{A_{n}}^{\sigma}\left(f \log \frac{f}{\mu_{A_{n}}^{\bullet}(f)}\right) \leq s\left(\mathcal{L}_{A_{n}}^{\sigma}\right)^{-1} \mathcal{E}_{A_{n}}^{\sigma}(f, \log f)
$$

and the same for the set $B_{n}$. So from (5.9) we obtain

$$
\begin{aligned}
\mu_{R}^{\tau}(f \log f) \leq & {\left[\inf _{\sigma \in \Omega} s\left(\mathcal{L}_{A_{n}}^{\sigma}\right) \wedge s\left(\mathcal{L}_{B_{n}}^{\sigma}\right)\right]^{-1} \mu_{R}^{\tau}\left[\mathcal{E}_{\dot{A}_{n}}(f, \log f)+\mathcal{E}_{B_{n}}(f, \log f)\right] } \\
& +2 e^{-C \sqrt{L}} \mu_{R}^{\tau}(f \log f) .
\end{aligned}
$$

Thus

$$
\mu_{R}^{\tau}(f \log f) \leq \gamma\left(L, A_{n}, B_{n}\right)\left[\mathcal{E}_{R}^{\tau}(f, \log f)+\sum_{x \in A_{n} \cap B_{n}} \mu_{R}^{\tau}\left(c_{R}^{\tau}(x, \cdot,+)\left|\nabla_{x}^{+} f\right|^{2}\right)\right],
$$

where

$$
\gamma\left(L, A_{n}, B_{n}\right)=\frac{1}{1-2 e^{-C \sqrt{L}}}\left[\inf _{\sigma \in \Omega} s\left(\mathcal{L}_{A_{n}}^{\sigma}\right) \wedge s\left(\mathcal{L}_{B_{n}}^{\sigma}\right)\right]^{-1} .
$$

After averaging over $n$ we obtain

$$
s\left(\mathcal{L}_{R}^{\tau}\right) \geq\left(1-\frac{C}{\sqrt{L}}\right) \min _{n} \inf _{\sigma \in \Omega}\left[s\left(\mathcal{L}_{A_{n}}^{\sigma}\right) \wedge s\left(\mathcal{L}_{B_{n}}^{\sigma}\right)\right] .
$$

The rest of the proof is identical to the proof of Proposition 4.1. 
Acknowledgment. We thank F. Cesi for teaching us inequality (5.7), which avoids the use of the spectral gap in the proof of the entropy inequality.

\section{REFERENCES}

[1] An, C., Blachre, S., Chafaï, D., Fougres, P., Gentil, I., Malrieu, F., Roberto, C. and Scheffer, G. (2000). Sur les ingalits de Sobolev logarithmiques. Panor. Synth. 10.

[2] Bertini, L., CAncrini, N. and Cesi, F. (2000). The spectral gap for a Glauber-type dynamics in a continuous gas. Ann. Inst. H. Poincaré Probab. Statist. To appear.

[3] BobKov, S. G. and Ledoux, M. (1998). On modified logarithmic Sobolev inequalities for Bernoulli and Poisson measures. J. Funct. Anal. 156 347-365.

[4] CESI, F. (2001). Quasi-factorization of the entropy and logarithmic Sobolev inequalities for Gibbs random fields. Probab. Theory Related Fields 120 569-584.

[5] Diaconis, P. and SAloff-Coste, L. (1996). Logarithmic Sobolev inequalities for finite Markov chains. Ann. Appl. Probab. 6 695-750.

[6] Dobrushin, R. L. and Shlosman, S. B. (1985). Constructive criterion for the uniqueness of Gibbs field. In Statistical Physics and Dynamical Systems 347-370. Birkhäuser, Boston.

[7] Gross, L. (1993). Logarithmic Sobolev inequalities and contractivity properties of semigroups. Dirichlet Forms. Lecture Notes in Math. 1563 54-88. Springer, Berlin.

[8] LAwler, G. F. and SokAL, A. D. (1988). Bounds on the $L^{2}$ spectrum for Markov chains and Markov processes: A generalization of Cheeger's inequality. Trans. Amer. Math. Soc. 309 557-580.

[9] Martinelli, F. (1999). Lectures on Glauber dynamics for discrete spin models. Lectures on Probability Theory and Statistics. Lecture Notes in Math. 1717 93-191. Springer, Berlin.

[10] Martinelli, F. and Olivieri, E. (1994). Approach to equilibrium of Glauber dynamics in the one phase region. II. The general case. Comm. Math. Phys. 161 487-514.

[11] Miclo, L. (1999). An example of application of discrete Hardy inequalities. Markov Process. Related Fields 5 319-330.

[12] Miclo, L. and Roberto, C. (2001). Modified logarithmic Sobolev inequality and Hardy type inequalities. Preprint.

[13] Stroock, D. W. and Zegarlinski, B. (1992). The logarithmic Sobolev inequality for discrete spin systems on a lattice. Comm. Math. Phys. 149 175-193.

[14] Stroock, D. W. and Zegarlinski, B. (1992). The logarithmic Sobolev inequality for continuous spin systems on a lattice. J. Funct. Anal. 104 299-326.

DiPARTIMENTO Di MATEMATICA

POLITECNICO DI MILANO

PiAZZA L. DA VinCI 32

20133 MILANO

ITALY

E-MAIL: daipra@math.unipd.it 